Portland State University

PDXScholar

8-1-2008

\title{
Green dyadic for the Proca fields
}

P.T. Leung

Portland State University

Paul Dragulin

Follow this and additional works at: https://pdxscholar.library.pdx.edu/phy_fac

Part of the Physics Commons

Let us know how access to this document benefits you.

\section{Citation Details}

Dragulin, P., \& Leung, P. (2008). Green dyadic for the Proca fields. Physical Review. E, Statistical, Nonlinear, And Soft Matter Physics, 78(2 Pt 2), 026605.

This Article is brought to you for free and open access. It has been accepted for inclusion in Physics Faculty Publications and Presentations by an authorized administrator of PDXScholar. Please contact us if we can make this document more accessible: pdxscholar@pdx.edu. 


\title{
Green dyadic for the Proca fields
}

\author{
Paul Dragulin and P. T. Leung (梁 培 德) ${ }^{*}$ \\ Department of Physics, Portland State University, P. O. Box 751, Portland, Oregon 97207-0751, USA
}

(Received 14 March 2008; published 14 August 2008)

\begin{abstract}
The dyadic Green functions for the Proca fields in free space are derived to include singular terms. Both the electric and magnetic types will be obtained with the results reduced back to those for the Maxwell fields in the limit of zero photon mass. Moreover, the singular terms are identical in both massless and massive electrodynamics. As an illustration, the results are applied to obtain the exact dynamical fields for an oscillating dipole which reduce back to the well-known expressions for static fields derived previously in the literature for massive electrodynamics.
\end{abstract}

DOI: 10.1103/PhysRevE.78.026605

PACS number(s): 03.50.De, 02.90.+p, 04.30.Nk

\section{INTRODUCTION}

Although the two fundamental forces-electromagnetic and gravitational-which govern most of the physical phenomena at a macroscopic scale are believed to be "truly long range" in nature, a nonvanishing value for either the photon mass or the graviton mass will have many significant implications from the dispersion of light in vacuum to certain fundamental issues in cosmology [1]. In particular, the possibility of having a finite photon mass has been studied intensively for almost a century, with both serious theoretical and experimental investigations devoted to it [2].

Theoretically, the simplest Lorentz invariant generalization of Maxwell's theory to include a finite photon mass was first worked out by Proca [3]; and the quantization of the Proca field is also possible despite the loss of gauge invariance in "massive electrodynamics" [2]. At the classical level, the Proca equations lead to modifications to only the two Maxwell equations with source, and the consequences of these have been studied by many researchers in the literature to include problems such as effects on special relativity, multipole radiations from localized sources, etc. Experimentally, the two main approaches to set an upper limit for the photon mass have been based on examination of the accuracy of Coulomb's law (static approach) and the dispersion of light in vacuum (dynamic approach). While the static approach can usually set more stringent limits on the upper bound of the photon mass, the dynamic approach can enable one to go beyond terrestrial experiments so that possible dispersion can be studied with light propagating over a large scale of distances. To date, one of the best upper limits established is believed to be in the order of $10^{-49} \mathrm{~g}$ for the mass of the photon [2].

Our interest here in the present work is to derive the most general expressions for the Green dyadic functions of the classical Proca field equations to include the singular terms in the dyadic. As is well known in conventional Maxwellian electrodynamics, the knowledge of such dyadic functions will allow one to calculate the general time-dependent fields

\footnotetext{
*Author to whom correspondence should be addressed. Present address: Dept. of Physics, National Taiwan University, Taipei 106, Taiwan.
}

for any arbitrary localized current sources [4]. New results for the dyadic Green functions are always significant whenever a new vector field theory is introduced [5]. As for the Proca field, previous work in the literature had derived the scalar Green function for the Proca wave equation. The results obtained had then been applied to the analysis of the radiations from binary pulsar, leading to new limits on the electric charge of various astrophysical bodies as well as on the strength of possible new forces of weak interaction [6]. However, neither the dyadic nor the singular term for the Proca field has been established in the previous studies. As for the latter, it has been recognized from time to time the importance of treating the singular behaviors of various fields and sources to the extent that a complete text has been written for the treatment of this problem [7]. For example, for the Maxwell field, both electrostatic [8] and electrodynamic dipole fields have been obtained with the correct singular terms included [9,10]. More generally, the Green dyadic (electric type) with the correct singularity has also been previously derived in the literature $[9,11]$. Here we shall derive in the following both the electric and magnetic dyadic functions for the Proca fields to include all the appropriate singular terms. We shall then apply these general results to derive the dynamical electric and magnetic fields from dipole sources according to the Proca theory, and recover some well-known results established in the literature under certain limiting conditions.

\section{GENERAL DYADIC FORMULATION}

Let us begin by recapitulating the following well-known Maxwell-Proca equations in vacuum (in Gaussian units):

$$
\begin{gathered}
\boldsymbol{\nabla} \cdot \mathbf{E}=4 \pi \rho-\mu^{2} \phi, \\
\boldsymbol{\nabla} \cdot \mathbf{B}=0, \\
\boldsymbol{\nabla} \times \mathbf{E}+\frac{1}{c} \frac{\partial \mathbf{B}}{\partial t}=\mathbf{0}, \\
\boldsymbol{\nabla} \times \mathbf{B}-\frac{1}{c} \frac{\partial \mathbf{E}}{\partial t}=\frac{4 \pi}{c} \mathbf{J}-\mu^{2} \mathbf{A},
\end{gathered}
$$

where $\mu=\frac{m c}{\hbar}$ is the inverse Compton wavelength of the photon with $m$ the photon mass and the fields are given via the 
usual expressions in terms of the potentials as revealed from Eqs. (2) and (3) as follows:

$$
\begin{gathered}
\mathbf{B}=\boldsymbol{\nabla} \times \mathbf{A}, \\
\mathbf{E}=-\boldsymbol{\nabla} \phi-\frac{1}{c} \frac{\partial \mathbf{A}}{\partial t} .
\end{gathered}
$$

As is well known [2], Eqs. (1) and (4) imply that the Lorentz gauge condition must be implemented so that the conservation of charge remains valid. In addition, it has been thoroughly studied in the literature [6] for the electromagnetic waves and radiations via the derivation of the following wave equations for the potentials:

$$
\begin{aligned}
& \left(\nabla^{2}-\frac{1}{c^{2}} \frac{\partial^{2}}{\partial t^{2}}-\mu^{2}\right) \phi=-4 \pi \rho, \\
& \left(\nabla^{2}-\frac{1}{c^{2}} \frac{\partial^{2}}{\partial t^{2}}-\mu^{2}\right) \mathbf{A}=-\frac{4 \pi \mathbf{J}}{c} .
\end{aligned}
$$

For harmonic sources and fields (i.e., $\sim e^{-i \omega t}$ ), these reduce to the Helmholtz equations with the well-known retarded Green's function given by

$$
G_{0}\left(\mathbf{r}, \mathbf{r}^{\prime}\right)=\frac{e^{i \beta\left|\mathbf{r}-\mathbf{r}^{\prime}\right|}}{\left|\mathbf{r}-\mathbf{r}^{\prime}\right|},
$$

where we have introduced an "effective wave number"

$$
\beta=\sqrt{\left(\frac{\omega}{c}\right)^{2}-\mu^{2}} \equiv \sqrt{k^{2}-\mu^{2}}
$$

and $G_{0}$ satisfies

$$
\left(\nabla^{2}+\beta^{2}\right) G_{0}\left(\mathbf{r}, \mathbf{r}^{\prime}\right)=-\delta\left(\mathbf{r}-\mathbf{r}^{\prime}\right) .
$$

Based on the introduction of this effective wave number together with the appropriate modifications of the various quantities such as the energy-momentum and Poynting vector for the Proca field, many results calculated in Maxwell (massless) electrodynamics for the radiation problem can be translated over to the case of "massive" electrodynamics [6]. However, in spite of having many of these previous results established in the literature, it seems that the problem of the Green dyadic and its singular behavior for the Proca field have not been studied in most of the previous works. While the same problem has been studied extensively in the case of conventional (massless) electrodynamics [4,9], it has been known from these studies that the result for the singularity of the fields is significant which not only provides consistency with the field equations, but also leads to interesting applications $[8,10]$. Furthermore, the knowledge of the Green dyadics enables one to calculate the electromagnetic fields directly from a given source without having to resort to the potentials. Hence we feel justified to present an explicit derivation for them and illustrate their applications to obtain new results for dipole fields which recover some well-known results for the Proca fields available in the literature.

To derive the Green dyadics, we have first to obtain the vector wave equations for the electric and magnetic fields just as in the case with the ordinary Maxwellian electrody- namics [4]. These can be obtained in a straightforward way from Eqs. (1)-(4) in the following form:

$$
\boldsymbol{\nabla} \times \boldsymbol{\nabla} \times \mathbf{E}-k^{2} \mathbf{E}+i k \mu^{2} \mathbf{A}=\frac{4 \pi i k}{c} \mathbf{J}
$$

and

$$
\boldsymbol{\nabla} \times \boldsymbol{\nabla} \times \mathbf{B}-\beta^{2} \mathbf{B}=\frac{4 \pi}{c} \boldsymbol{\nabla} \times \mathbf{J} .
$$

Hence we see that while the equation for the magnetic field (with the use of the "effective wave number") has the same form as that in Maxwell's electrodynamics, the one for the electric field is different and has the vector potential appeared explicitly. However, we shall see below that this will not affect at all the usual way for the determination of the dyadics in terms of the scalar Green's function in Eq. (9). To show explicitly this is the case, let us introduce the electric $\left(\boldsymbol{G}_{e}\right)$ and magnetic $\left(\boldsymbol{G}_{m}\right)$ dyadic Green function for the Proca field by making the following ansatz:

$$
\boldsymbol{\nabla} \times \boldsymbol{\nabla} \times \boldsymbol{G}_{e}-k^{2} \boldsymbol{G}_{e}+\mu^{2} \boldsymbol{I} G_{0}=\boldsymbol{I} \delta\left(\mathbf{r}-\mathbf{r}^{\prime}\right)
$$

and

$$
\boldsymbol{\nabla} \times \boldsymbol{\nabla} \times \boldsymbol{G}_{m}-\beta^{2} \boldsymbol{G}_{m}=\boldsymbol{\nabla} \times\left[\boldsymbol{I} \delta\left(\mathbf{r}-\mathbf{r}^{\prime}\right)\right],
$$

where $\boldsymbol{I}$ is the unit (identity) dyadic and $G_{0}$ is as given in Eq. (9). Note that while Eq. (15) is completely analogous to that for the Maxwell field, the result in Eq. (14) is unique for the Proca field which mixes the electric dyadic Green function with the scalar Green function (introduced for solving the vector potential) through the mass term $\left(\sim \mu^{2}\right)$. Although the form of Eq. (14) is strongly suggested from that of Eq. (12) together with the results in Eqs. (8)-(11) - by noting that each $\boldsymbol{G}_{e}$ and $G_{0}$ are simply related to the corresponding electric field and vector potential for a point source-it is indeed possible to show explicitly the consistency of this new scheme of introducing the electric Green dyadic, with the conventional determination of the dyadics in terms of the scalar Green function as in Maxwell electrodynamics [4].

To begin, we first note that since Eqs. (2), (3), (5), and (6) are identical to those in Maxwell electrodynamics, we have therefore the well-known relations between the dyadics and the scalar Green function $G_{0}$ as follows [4]:

$$
\begin{gathered}
\boldsymbol{G}_{e}=\left(\boldsymbol{I}+\frac{1}{k^{2}} \boldsymbol{\nabla} \boldsymbol{\nabla}\right) G_{0}, \\
\boldsymbol{G}_{m}=\boldsymbol{\nabla} \times\left(\boldsymbol{I} G_{0}\right) .
\end{gathered}
$$

Next we show that the result in Eq. (16) is indeed a solution to Eq. (14) with the help of the wave equation in Eq. (11). Thus we have from Eq. (16)

$$
\begin{aligned}
\boldsymbol{\nabla} \times \boldsymbol{\nabla} \times \boldsymbol{G}_{e}-k^{2} \boldsymbol{G}_{e} & =\boldsymbol{\nabla} \times \boldsymbol{\nabla} \times \boldsymbol{I} G_{0}-k^{2} \boldsymbol{G}_{e} \\
& =\boldsymbol{\nabla}\left(\boldsymbol{\nabla} \cdot \boldsymbol{I} G_{0}\right)-\nabla^{2} \boldsymbol{I} G_{0}-k^{2} \boldsymbol{I} G_{0}-\boldsymbol{\nabla} \boldsymbol{\nabla} G_{0} \\
& =-\boldsymbol{I}\left(\nabla^{2}+k^{2}\right) G_{0}
\end{aligned}
$$

since the second term in Eq. (16) is curless, and the first and last terms cancel in the second row in Eq. (18). On the other hand, we have from Eq. (11) 


$$
\begin{aligned}
\left(\nabla^{2}+k^{2}\right) G_{0}\left(\mathbf{r}, \mathbf{r}^{\prime}\right) & =\left(\nabla^{2}+\beta^{2}+\mu^{2}\right) G_{0}\left(\mathbf{r}, \mathbf{r}^{\prime}\right) \\
& =-\delta\left(\mathbf{r}-\mathbf{r}^{\prime}\right)+\mu^{2} G_{0}\left(\mathbf{r}, \mathbf{r}^{\prime}\right) .
\end{aligned}
$$

Substitution of Eq. (19) into Eq. (18) leads immediately to the result in Eq. (14). Hence the result in Eq. (14), which mixes the scalar and dyadic Green functions, indeed provides a consistent scheme for the introduction of $G_{e}$ in the case of massive electrodynamics, and is determined the usual way via Eq. (16). Once this is established, the dyadics introduced in Eqs. (14) and (15) will be the correct dyadics for the Proca fields through the uniqueness theorem for Green functions. With the dyadics given in Eqs. (16) and (17), the fields can then be obtained directly in terms of the source current in the following forms:

$$
\mathbf{E}(\mathbf{r})=\frac{4 \pi i k}{c} \int \boldsymbol{G}_{e}\left(\mathbf{r}, \mathbf{r}^{\prime}\right) \mathbf{J}\left(\mathbf{r}^{\prime}\right) d^{3} x^{\prime}
$$

and

$$
\mathbf{B}(\mathbf{r})=\frac{4 \pi}{c} \int \boldsymbol{G}_{m}\left(\mathbf{r}, \mathbf{r}^{\prime}\right) \mathbf{J}\left(\mathbf{r}^{\prime}\right) d^{3} x^{\prime}
$$

\section{CALCULATION OF GREEN DYADIC}

In this section, we show how the dyadics in Eqs. (16) and (17) can be calculated with the singular terms explicitly included. For the case of the Maxwell fields, the singularity of the electric dyadic $\boldsymbol{G}_{e}$ has been obtained previously in the literature $[4,9,11]$. Here we shall follow a different approach by deriving both $\boldsymbol{G}_{e}$ and $\boldsymbol{G}_{m}$ via a direct calculation, with the application of the various differentiation identities for the $(1 / r)$ potential function involving the $\delta$ function which are well established in the literature [12].

For simplicity, let us set $\mathbf{r}^{\prime}=\mathbf{0}$ and consider a component of the dyadic tensor. Thus from Eqs. (9) and (16), we have

$$
\begin{aligned}
\left(\boldsymbol{G}_{e}\right)_{p q} & =\left(\delta_{p q}+\frac{1}{k^{2}} \partial_{p} \partial_{q}\right)\left(\frac{e^{i \beta r}}{4 \pi r}\right) \\
& =\frac{e^{i \beta r}}{4 \pi r} \delta_{p q}+\frac{1}{4 \pi k^{2}}\left(\frac{1}{r} \partial_{p} \partial_{q} e^{i \beta r}+\partial_{p} \frac{1}{r} \partial_{q} e^{i \beta r}+\partial_{q} \frac{1}{r} \partial_{p} e^{i \beta r}+e^{i \beta r} \partial_{p} \partial_{q} \frac{1}{r}\right) \\
& =\frac{e^{i \beta r}}{4 \pi r} \delta_{p q}+\frac{e^{i \beta r}}{4 \pi k^{2}}\left(-\frac{1}{r^{3}} \beta^{2} x_{p} x_{q}+\frac{i \beta}{r^{2}} \delta_{p q}-i \beta \frac{3 x_{p} x_{q}}{r^{4}}-\frac{4 \pi}{3} \delta(\mathbf{r}) \delta_{p q}+\frac{3 x_{p} x_{q}-r^{2} \delta_{p q}}{r^{5}}\right) \\
& =-\frac{1}{3 k^{2}} \delta(\mathbf{r}) \delta_{p q}+\frac{e^{i \beta r}}{4 \pi k^{2} r^{5}}\left[r^{2}\left(-1+i \beta r+k^{2} r^{2}\right) \delta_{p q}+\left(3-3 i \beta r-\beta^{2} r^{2}\right) x_{p} x_{q}\right],
\end{aligned}
$$

where we have used the identity $\partial_{p} \partial_{q}(1 / r)$ $=-(4 \pi / 3) \delta_{p q} \delta(\mathbf{r})+\left(3 x_{p} x_{q}-r^{2} \delta_{p q}\right) / r^{5}$ established in Ref. [12]. We also note that the singular term will be the same as that for the case of static field $(k \rightarrow 0)$ and/or massless photon $(\mu \rightarrow 0)$, for the term $e^{i \beta r} \delta(\mathbf{r})$ is actually equivalent to $\delta(\mathbf{r})$ since $e^{i \beta r}=1$ at $r=0$. Thus the general form of the electric dyadic for the Proca field including the singularity term can be finally obtained in the following form:

$$
\begin{aligned}
\boldsymbol{G}_{e}\left(\mathbf{r}, \mathbf{r}^{\prime}\right)= & -\frac{1}{3 k^{2}} \delta\left(\mathbf{r}-\mathbf{r}^{\prime}\right) \boldsymbol{I}+\frac{e^{i \beta\left|\mathbf{r}-\mathbf{r}^{\prime}\right|}}{4 \pi k^{2}\left|\mathbf{r}-\mathbf{r}^{\prime}\right|^{5}}\left[\left|\mathbf{r}-\mathbf{r}^{\prime}\right|^{2}\right. \\
& \times\left(-1+i \beta\left|\mathbf{r}-\mathbf{r}^{\prime}\right|+k^{2}\left|\mathbf{r}-\mathbf{r}^{\prime}\right|^{2}\right) \boldsymbol{I} \\
& \left.+\left(3-3 i \beta\left|\mathbf{r}-\mathbf{r}^{\prime}\right|-\beta^{2}\left|\mathbf{r}-\mathbf{r}^{\prime}\right|^{2}\right)\left(\mathbf{r}-\mathbf{r}^{\prime}\right)\left(\mathbf{r}-\mathbf{r}^{\prime}\right)\right]
\end{aligned}
$$

Note that Eq. (23) can be applied to calculate the dynamical electric field from an arbitrary localized source in massive electrodynamics. In the limit of massless Maxwell electrodynamics, $\beta \rightarrow k$ and the result in Eq. (23) reproduces the previous result obtained in the literature [9].

Next we derive the corresponding magnetic dyadic for the Proca field. The possible singularity in this case has not been clarified previously even for the massless Maxwell case [9], but it is rather straightforward and we shall see that there are no explicit singular terms associated with it. However, singular terms can still emerge during the calculation of the magnetic fields using this dyadic (see below). Again, we set $\mathbf{r}^{\prime}=\mathbf{0}$ and apply Eq. (9) to a component of Eq. (17) as follows:

$$
\left(\boldsymbol{G}_{m}\right)_{p q}=\varepsilon_{p s t} \partial_{s}\left(\delta_{t q} G_{0}\right)=\varepsilon_{p s q} \partial_{s}\left(\frac{e^{i \beta r}}{4 \pi r}\right)
$$

so that the dyadic can finally be expressed in the following form

$$
\boldsymbol{G}_{m}\left(\mathbf{r}, \mathbf{r}^{\prime}\right)=\left(1-i \beta\left|\mathbf{r}-\mathbf{r}^{\prime}\right|\right) e^{i \beta\left|\mathbf{r}-\mathbf{r}^{\prime}\right|} \boldsymbol{I} \times \frac{\left(\mathbf{r}-\mathbf{r}^{\prime}\right)}{\left|\mathbf{r}-\mathbf{r}^{\prime}\right|^{3}} .
$$

\section{APPLICATION TO THE CALCULATION OF DIPOLE FIELDS}

As an illustration of the usefulness of the results in Eqs. (22)-(25), we shall apply them to Eqs. (20) and (21) to derive the fully dynamical dipole fields for massive electrodynamics according to the Proca equations. 


\section{A. Electric dipole fields}

For a harmonic oscillating electric dipole $\mathbf{p}$ at the origin, we have the current density given by $\mathbf{J}\left(\mathbf{r}^{\prime}\right)=-i \omega \mathbf{p} \delta\left(\mathbf{r}^{\prime}\right)[8]$. Thus from Eq. (20), we obtain the following expression for the electric field:

$$
\mathbf{E}(\mathbf{r})=4 \pi k^{2} \boldsymbol{G}_{e}(\mathbf{r}, \mathbf{0}) \mathbf{p},
$$

which, on using the result in Eq. (23) yields the following result for the electric dipole Proca field:

$$
\begin{aligned}
\mathbf{E}(\mathbf{r})= & -\frac{4 \pi}{3} \mathbf{p} \delta(\mathbf{r})+\frac{e^{i \beta r}}{r^{5}}\left[r^{2}\left(-1+i \beta r+k^{2} r^{2}\right) \mathbf{p}\right. \\
& \left.+\left(3-3 i \beta r-\beta^{2} r^{2}\right)(\mathbf{r} \cdot \mathbf{p}) \mathbf{r}\right] .
\end{aligned}
$$

Let us examine the various limiting cases derived from Eq. (27). For the case of "massive electrostatics," we have $k$ $\rightarrow 0$ and $\beta^{2} \rightarrow-\mu^{2}$, Eq. (27) yields the following electrostatic Yukawa field:

$$
\begin{aligned}
\mathbf{E}(\mathbf{r})= & -\frac{4 \pi}{3} \mathbf{p} \delta(\mathbf{r})+\frac{e^{-\mu r}}{r^{5}}\left[r^{2}(-1-\mu r) \mathbf{p}\right. \\
& \left.+\left(3+3 \mu r+\mu^{2} r^{2}\right)(\mathbf{r} \cdot \mathbf{p}) \mathbf{r}\right]
\end{aligned}
$$

which was obtained previously in the literature [13] (except for the singular term and a small error). It is clear that in the case of zero photon mass $\mu=0$ and Eq. (28) reduces back to the well-known electric dipole field with the singular term as can be found in standard electrodynamics texts [8]. On the other hand, if we set $\mu=0$ in Eq. (27), we will recover the following well-known dynamical electric dipole field [8] together with the same singular term as for the static field $[9,10]$ :

$$
\begin{aligned}
\mathbf{E}(\mathbf{r})= & -\frac{4 \pi}{3} \mathbf{p} \delta(\mathbf{r})+\frac{e^{i k r}}{r^{5}}\left[r^{2}\left(-1+i k r+k^{2} r^{2}\right) \mathbf{p}\right. \\
& \left.+\left(3-3 i k r-k^{2} r^{2}\right)(\mathbf{r} \cdot \mathbf{p}) \mathbf{r}\right]
\end{aligned}
$$

\section{B. Magnetic dipole field}

For a magnetic dipole $\overrightarrow{\mathbf{m}}$ at the origin, we have the current density given by $\mathbf{J}\left(\mathbf{r}^{\prime}\right)=c \boldsymbol{\nabla}^{\prime} \times \mathbf{M}=c \boldsymbol{\nabla}^{\prime} \times\left[\mathbf{m} \delta\left(\mathbf{r}^{\prime}\right)\right]$. To calculate the magnetic field due to this current, it is safer if we go back to calculate one of its component using the result in Eq. (24) so that we will not miss any possible singular terms from differentiating twice the potential function $(1 / r)$ [12]. Thus we have

$$
B_{p}(\mathbf{r})=\frac{4 \pi}{c} \int \varepsilon_{p s q} \partial_{s}\left(\frac{e^{i \beta\left|\mathbf{r}-\mathbf{r}^{\prime}\right|}}{4 \pi\left|\mathbf{r}-\mathbf{r}^{\prime}\right|}\right) \cdot c \varepsilon_{q a b} \partial_{a}^{\prime}\left[m_{b} \delta\left(\mathbf{r}^{\prime}\right)\right] d^{3} x^{\prime} .
$$

By converting $\partial_{s}$ to $-\partial_{s}^{\prime}$ and integrating by parts, Eq. (30) can be evaluated to obtain

$$
B_{p}(\mathbf{r})=\left(\delta_{p a} \delta_{s b}-\delta_{p b} \delta_{s a}\right) m_{b} \int \partial_{a}^{\prime} \partial_{s}^{\prime}\left(\frac{e^{i \beta\left|\mathbf{r}-\mathbf{r}^{\prime}\right|}}{\left|\mathbf{r}-\mathbf{r}^{\prime}\right|}\right) \delta\left(\mathbf{r}^{\prime}\right) d^{3} x^{\prime}
$$

The evaluation of the double derivative in the integrand has already been calculated before in deriving Eq. (22). Using the result obtained there and integrating over the delta function in Eq. (31), we finally obtain

$$
\begin{aligned}
B_{p}(\mathbf{r})= & \left(\delta_{p a} \delta_{s b}-\delta_{p b} \delta_{s a}\right) m_{b} e^{i \beta r}\left(-\frac{1}{r^{3}} \beta^{2} x_{a} x_{s}+\frac{i \beta}{r^{2}} \delta_{a s}\right. \\
& \left.-i \beta \frac{3 x_{a} x_{s}}{r^{4}}-\frac{4 \pi}{3} \delta(\mathbf{r}) \delta_{a s}+\frac{3 x_{a} x_{s}-r^{2} \delta_{a s}}{r^{5}}\right) \\
= & e^{i \beta r}\left[\frac{8 \pi}{3} \delta(\mathbf{r}) m_{p}+\left(-\frac{1}{r^{3}}+\frac{i \beta}{r^{2}}+\frac{\beta^{2}}{r}\right) m_{p}\right. \\
& \left.+\left(\frac{3}{r^{5}}-\frac{3 i \beta}{r^{4}}-\frac{\beta^{2}}{r^{3}}\right)(\mathbf{r} \cdot \mathbf{m}) x_{p}\right],
\end{aligned}
$$

which leads to the following expression for the dynamical Proca field due to a harmonically oscillating magnetic dipole at the origin:

$$
\begin{aligned}
\mathbf{B}(\mathbf{r})= & \frac{8 \pi}{3} \mathbf{m} \delta(\mathbf{r})+\frac{e^{i \beta r}}{r^{5}}\left[r^{2}\left(-1+i \beta r+\beta^{2} r^{2}\right) \mathbf{m}\right. \\
& \left.+\left(3-3 i \beta r-\beta^{2} r^{2}\right)(\mathbf{r} \cdot \mathbf{m}) \mathbf{r}\right] .
\end{aligned}
$$

Note that Eq. (33) is a generalization of a result first obtained by Schrödinger [14] for the static case to the dynamical case with the explicit inclusion of the singular term. Again, one can check all the required limits as follows. For example, by setting $k \rightarrow 0$ and $\beta^{2} \rightarrow-\mu^{2}$, one recovers the magnetostatic result first obtained by Schrödinger [14]:

$$
\begin{aligned}
\mathbf{B}(\mathbf{r})= & \frac{8 \pi}{3} \mathbf{m} \delta(\mathbf{r})+\frac{e^{-\mu r}}{r^{5}}\left[-r^{2}\left(1+\mu r+\mu^{2} r^{2}\right) \mathbf{m}\right. \\
& \left.+\left(3+3 \mu r+\mu^{2} r^{2}\right)(\mathbf{r} \cdot \mathbf{m}) \mathbf{r}\right],
\end{aligned}
$$

with the singular term included. In the limit of zero photon mass, Eq. (34) reduces back to the well-known expression obtained by Jackson [8]. On the other hand, by setting the mass to zero in Eq. (33), $\beta \rightarrow k$ and one recovers the dynamic magnetic dipole field in Maxwell's theory with the singular term explicitly included [10].

\section{DISCUSSION AND CONCLUSION}

In this work, we have derived the dyadic Green functions for "massive electrodynamics" by using Proca's equations to include the singular term in the electric dyadic, while none is needed for the magnetic dyadic [Eqs. (22)-(25)]. As in conventional electrodynamics, the knowledge of these dyadics is very useful for they enable one to calculate the electromagnetic fields directly from any given localized source [4], especially when these sources are expressed in multipole forms [15]. As an illustration, we have worked out in details both the electric and magnetic dipole fields, which reduce to the appropriate results well established in the literature in the 
case of statics and/or Maxwell (massless) electrodynamics. We also note that the singularity of the dyadic is unaffected by either the photon mass or the dynamic behavior of the fields. The physics behind this is that while the singular behavior is dominated by the near fields; the Yukawa factor $\left(e^{-\mu r}\right)$ becomes unity at the location of the source in the case of the Proca fields. However, we notice that, unlike the Maxwell case where the dipole electric and magnetic fields have exactly the same form away from the source, this symmetry is destroyed in the presence of the photon mass as can be seen from Eqs. (27) and (33). Such asymmetry arises since in the Proca theory, the mass term affects only the source pair of Maxwell's equations (1) and (4), while the other pair remains homogeneous and is not affected. This occurs due to the absence of magnetic monopoles and the associated currents even in the Proca theory [16], and it is the same origin for the difference in the two singular terms in Eqs. (27) and
(33) $[8,17]$. These singular terms can be of high significance in quantum physics since a charged particle in the form of a wave can have nonzero presence at the origin of the source for the fields. A famous example of these is the application of the $\delta$ function term in the magnetic dipole field to the calculation of the hyperfine transition for ground state $\mathrm{H}$ atom, leading to the famous $21 \mathrm{~cm}$ emission in astronomy [8]. It will be of interest to find further applications of the general results obtained for the Green dyadics in Eqs. (22)-(25) in our present work such as in the calculation of higher order multipole fields.

\section{ACKNOWLEDGMENTS}

One of us (P.T.L.) would like to thank Professor G.- J. Ni for useful discussions, and the late Madame Luk Han-Yin for her lifelong support.
[1] See, e.g., the recent proposal of the possibility to have massive graviton as a candidate for cold-dark-matter in the Universe by S. L. Dubovsky, P. G. Tinyakov, and I. I. Tkachev, Phys. Rev. Lett. 94, 181102 (2005).

[2] See the recent very comprehensive review by L.-C. Tu, J. Luo, and G. T. Gillies, Rep. Prog. Phys. 68, 77 (2005).

[3] A. Proca, Compt. Rend. 190, 1377 (1930); many other original references by Proca can be found from Ref. [2] above.

[4] C.-T. Tai, Dyadic Green Function in Electromagnetic Theory (IEEE, New York, 1994).

[5] As an example in the literature, such dyadic was derived for the Beltrami-Maxwell fields in a general material continuum by W. S. Weiglhofer and A. Lakhtakia, in Phys. Rev. E 49, 5722 (1994).

[6] D. E. Krause, H. T. Kloor, and E. Fischbach, Phys. Rev. D 49, 6892 (1994).

[7] J. V. Bladel, Singular Electromagnetic Fields and Sources (Clarendon Press, Oxford, 1991).
[8] J. D. Jackson, Classical Electrodynamics (Wiley, New York, 1998).

[9] W. Weiglhofer, Am. J. Phys. 57, 455 (1989).

[10] P. T. Leung, Eur. J. Phys. 29, 137 (2008).

[11] See also, V. M. Finkel'berg, Sov. Phys. Tech. Phys. 9, 396 (1964); Y. A. Ryzhov, Sov. Phys. JETP 21, 433 (1965); and C. T. Tai, Proc. IEEE 61, 480 (1973).

[12] C. P. Frahm, Am. J. Phys. 51, 826 (1983).

[13] G. Omanovic, Found. Phys. Lett. 10, 427 (1997).

[14] E. Schrodinger, Proc. R. Ir. Acad., Sect. A 49, 43 (1943); 49, 135 (1943)

[15] E. G. P. Rowe, J. Phys. A 12, 245 (1979).

[16] There is some discussion in the literature on the incompatibility of a finite photon mass with the existence of magnetic monopole. See, e.g., A. Y. Ignatiev and G. C. Joshi, Mod. Phys. Lett. A 11, 2735 (1996).

[17] P. T. Leung and G. J. Ni, Eur. J. Phys. 27, N1 (2006). 